UVX 2010 (2011) 107-113

DOI: $10.1051 / \mathrm{uvx} / 2011015$

(C) Owned by the authors, published by EDP Sciences, 2011

\title{
Réalisation et caractérisation de photodétecteurs UV par laser à excimère (ArF)
}

\author{
Y. Larmande ${ }^{1, *}$, V. Vervisch ${ }^{1}$, Ph. Delaporte ${ }^{1}$, T. Sarnet $^{1}$, M. Sentis ${ }^{1}$, \\ H. Etienne ${ }^{2}$, F. Torregrosa ${ }^{2}$ et L. Roux ${ }^{2}$ \\ ${ }^{1}$ Laboratoire LP3, Parc scientifique et technologique de Luminy, 163 Av. de Luminy, C.917, \\ 13288 Marseille Cedex 9, France \\ 2 Ion Beam Services, ZI Peynier-Rousset, Rue Gaston Imbert prolongée, 13790 Peynier, \\ France
}

\begin{abstract}
Résumé. Dans le domaine de la microélectronique, la diminution de la taille des composants est un point clé du développement industriel. La réalisation de jonctions ultra-minces (USJ : Ultra-Shallow Junctions) est devenue une alternative évidente pour les futures technologies CMOS (Complementary Metal Oxide Semiconductor). Elle présente également un fort intérêt pour d'autres applications telles que la conception de détecteurs UV. Dans ce domaine de longueurs d'onde, la pénétration des photons dans le silicium est inférieure á $10 \mathrm{~nm}$. La zone de charge d'espace devra se trouver en surface pour diminuer les pertes de signal par recombinaison.

Pour commencer, nous présenterons les performances atteintes par l'association d'une implantation par immersion plasma (PIII : Plasma Immersion Ions Implantation) [1-3] et d'un recuit au laser á excimère (ELA : Excimer Laser Annealing) [4-7]. A l'aide de caractérisations électriques par mesures quatre pointes, nous pouvons estimer le niveau d'activation de nos jonctions. Des analyses SIMS nous ont permis de mesurer le profil de concentration en dopants après activation. Les jonctions obtenues ont une résistance par carré inférieure á $500 \Omega /$ sq pour une profondeur de jonction de $23 \mathrm{~nm}$. Enfin, nous avons réalisé des mesures de courant de fuite sur diodes afin de caractériser la quantité de défauts dans la jonction.

Pour compléter cette étude, nous mettrons en évidence des inhomogénéités dues au traitement laser. Des cartographies du photo-courant (LBIC : Light Beam Induced Current) [8-11] á différentes longueurs d'onde nous ont permis de relier ces inhomogénéités aux bords du faisceau laser. Il a été possible par la suite de relier la génération des défauts á un profil non-abrupte de bord de faisceau. Afin de mettre en évidence des défauts dans la structure cristalline du silicium, nos jonctions ont été caractérisées par microscopie électronique á transmission (TEM), ce qui a permis de localiser les défauts sur une couche de $3 \mathrm{~nm}$ en surface du substrat.
\end{abstract}

\section{INTRODUCTION}

La miniaturisation des circuits intégrés nécessite l'élaboration de nouvelles techniques de dopage afin de réaliser les extensions source/drain. Les spécifications demandées par l'ITRS (International Technology Roadmap for Semiconductor) [12] en termes de profil de jonction et d'activation, pour les prochain nœuds technologiques (taille de la grille inférieure á $22 \mathrm{~nm}$ ) ne sont pas atteintes par les techniques de production classiques. D’un autre coté, la fabrication de détecteurs UV nécessite de réaliser des USJ avec un taux d'activation important. A cause de la forte absorption du silicium dans l'UV, les photoporteurs risquent de se recombiner avant d'atteindre la zone de charge d'espace.

*e-mail : larmande@lp3.univ-mrs.fr

This is an Open Access article distributed under the terms of the Creative Commons Attribution-Noncommercial License 3.0, which permits unrestricted use, distribution, and reproduction in any noncommercial medium, provided the original work is properly cited. 
Afin de réaliser des USJ, nous avons utilisé l'implantation ionique par immersion plasma [1-3]. Cette technique permet d'introduire des ions á de très faibles énergies, ce qui correspond á des profondeurs d'implantation de quelques nanomètres. Cette étape est suivie d'un recuit d'activation effectué á l'aide d'un laser á excimère de type ArF [4-7]. Ce laser émettant dans l'UV, son énergie est absorbée dans les premiers nanomètres, et sa courte durée d'impulsion permet de limiter la diffusion des dopants. Dans un premier temps, nous avons étudié les principales caractéristiques des jonctions obtenues : résistance par carré, profils d'implantation et courants de fuite. Dans la littérature, nous avons observé que l'association de l'implantation par immersion plasma et du recuit laser permettait de doper une couche très fine avec un niveau d'activation dépassant la solubilité limite du bore dans le silicium [13-15]. Par la suite nous nous sommes intéressés á l'homogénéité du traitement laser. En effet, la taille de la zone á traiter étant bien supérieure á la taille de l'impact laser, un balayage de la surface est donc nécessaire. Chaque impact laser va engendrer des gradients thermiques importants dans le silicium au niveau des bords de faisceau. Des analyses LBIC [8-11] nous permettent de mettre en évidence que les régions où la concentration en défauts est plus importante correspondent aux bords des impacts laser. Nous avons étudié les mécanismes á l'origine de ces inhomogénéités afin d'en réduire les effets.

\section{PROCÉDÉ EXPÉRIMENTAL}

Des substrats de silicium de type $\mathrm{n}$ ont été utilisés pour réaliser nos échantillons. L'implantation de bore a été effectuée sur PULSION ${ }^{\circledR}$, un implanteur ionique par immersion plasma développé par la société IBS, avec différentes tensions d'accélération : $0,5 \mathrm{kV}, 0,75 \mathrm{kV}$ et $1 \mathrm{kV}$. La dose associée á chaque implantation est la dose de saturation. La profondeur de jonction, déterminée pour une concentration de $10^{18} \mathrm{at} / \mathrm{cm}^{3}$, est inférieure á $16 \mathrm{~nm}$. Les échantillons ont ensuite été recuits á l'aide d'un laser á excimère de type $\operatorname{ArF}(193 \mathrm{~nm}, 15 \mathrm{~ns})$, á différentes densités d'énergie et différents nombres de tirs.

Les mesures de résistance par carré, utilisant la technique 4 pointes, ont permis d'estimer le taux d'activation du bore dans la jonction. Des profils de concentration en dopants ont été obtenus par analyse SIMS (Secondary Ions Mass Spectrometry). Grâce á ces derniers, nous pouvons mesurer la profondeur de jonction. Des mesures I(V) ont permis d'estimer les courants de fuite (pour une tension appliquée de $-10 \mathrm{~V})$, qui sont caractéristiques de la présence de défauts dans la jonction.

Afin d'étudier l'homogénéité du traitement, un banc de mesure LBIC a été développé avec différentes sources lumineuses. Cette spécificité permet ainsi de sonder la jonction á différentes profondeurs dans le substrat. Les cartographies de la mesure du photo-courant mettent en évidence des inhomogénéités de la réponse á l'excitation lumineuse, qui sont corrélées á la présence de défauts. Il a donc été possible, en utilisant cette technique de caractérisation, d'étudier les phénomènes pouvant induire ces variations de réponse en photo-courant. Pour finir, deux échantillons ont été découpés par FIB (Focused Ions Beam). Le premier sur un bord de faisceau et le second sur un centre d'impact. Les analyses TEM de ces échantillons nous ont permis d'étudier la structure cristalline de la couche fondue.

\section{RÉSULTATS}

Deux aspects de caractérisation sont abordés dans cet article. Le premier concerne les caractéristiques électriques et chimiques des jonction obtenues par des mesures locales. Le second est une caractérisation de l'uniformité du traitement sur la surface de l'échantillon. Pour étudier ce paramètre, des cartographies de la réponse en photo-courant ont été effectuées.

\subsection{Paramètres de jonctions}

La figure 1(a) présente l'effet de la densité d'énergie laser sur la résistance par carré pour différents implantations. Pour les 3 courbes, le comportement est identique : l'activation est insuffisante en dessous de $400 \mathrm{~mJ} . \mathrm{cm}^{-2}$ et chute brutalement pour atteindre un palier. On observe un effet de seuil 

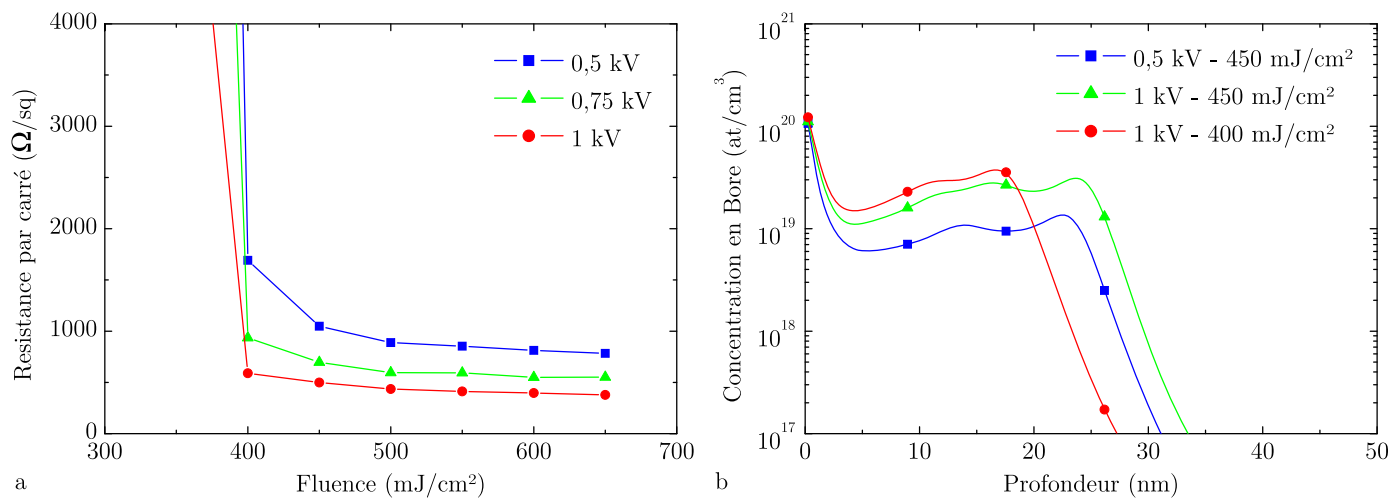

Figure 1. a) Résistance par carré en fonction de la densité d'énergie laser de recuit, pour différentes implantations b) Profils de concentration en dopants en fonction de la profondeur pour différentes conditions de dopage.

qui correspond á la densité d'énergie de fusion du silicium. Au-dessus de ce seuil, la résistance par carré n'évolue quasiment plus, ce qui signifie que l'activation est totale. Le fait que la résistance par carré á l'activation totale varie en fonction de la tension d'implantation est due á la dose de saturation. Cette dernière augmente avec la tension d'implantation, ce qui diminue la résistivité de la couche.

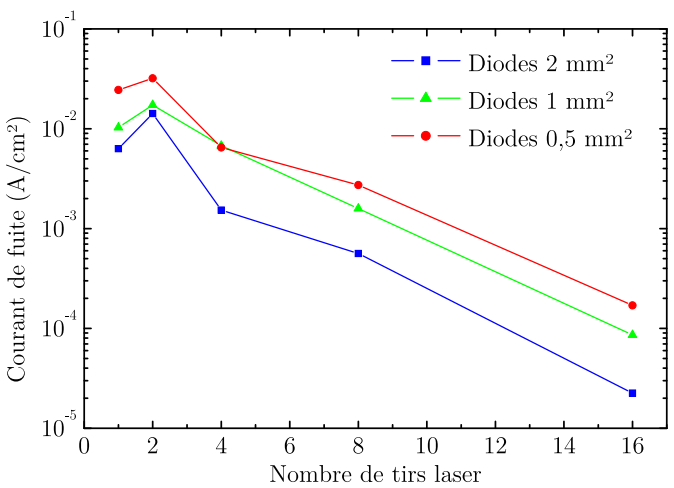

Figure 2. Densité de courant de fuite en fonction du nombre d'impacts laser de recuit pour différentes tailles de diode.

Des analyses SIMS ont été réalisées sur des échantillons recuits afin de mesurer la profondeur des jonctions. La figure 1(b) présente les profils de concentration en dopants en fonction de la profondeur, pour différentes conditions de dopage. Les comportements sont identiques : on remarque la présence d'un plateau dans la couche qui a été recuite, puis une chute brutale (moins de $3 \mathrm{~nm} / \mathrm{dec}$ ) au delá de la zone fondue. Ce type de profil est caractéristique du recuit laser. Pour une densité d'énergie donnée, la concentration en bore du plateau est nettement plus faible dans le cas d'une implantation á basse tension d'accélération, ce qui correspond au fait que la dose implantée diminue lorsque la tension d'implantation diminue. Cependant, la densité d'énergie laser imposant la profondeur de jonction, ces dernières prises á $10^{18}$ at.cm ${ }^{-3}$ sont très similaires pour les deux conditions d'implantation. Pour une implantation de $1 \mathrm{kV}$, on remarque sur la figure 1(a) que la résistance par carré évolue très peu entre 400 et $450 \mathrm{~mJ} . \mathrm{cm}^{-2}$ (valeurs respectives : 590 et $500 \Omega / \mathrm{sq}$ ) car l'on se trouve á des niveaux d'activation très élevés. Cependant, la profondeur de jonction passe de 23 á $29 \mathrm{~nm}$. Ainsi, la condition d'implantation á $1 \mathrm{kV}$ avec un recuit á $400 \mathrm{~mJ} . \mathrm{cm}^{-2}$ semble le meilleur compromis. 
Tableau 1. Propriétés optiques du silicium pour les sources d'analyse LBIC [16].

\begin{tabular}{llll}
\hline$\lambda(\mathrm{nm})$ & $\mathrm{E}_{\gamma}(\mathrm{eV})$ & $\alpha^{-1}(\mathrm{~nm})$ & $\mathrm{R}(\%)$ \\
\hline 193 & 6,44 & 5,6 & 68,8 \\
400 & 3,11 & 82 & 48,6 \\
633 & 1,96 & 2290 & 35,1 \\
800 & 1,55 & 7900 & 33,1 \\
\hline
\end{tabular}

Le dernier paramètre étudié pour caractériser nos jonctions est le courant de fuite. Ces mesures ont été effectuées sur des diodes de différentes tailles : $0,5,1$ et $2 \mathrm{~mm}^{2}$. Comme le montre la figure 2, la densité de courant de fuite est très élevée pour des activations réalisées avec un faible nombre de tirs laser, puis elle diminue lorsque l'on augmente le nombre de tirs. On peut supposer que les premiers impacts laser, qui placent les atomes dopants en sites substitutionnels, laissent une quantité de défauts importante. Les tirs suivants vont alors corriger les défauts restants.

\subsection{Homogénéité du traitement laser}

Nous avons donc obtenu des jonctions avec de bonnes propriétés en terme de niveaux d'activation et de profils de concentration en dopant. Cependant, les courants de fuite mesurés restent assez élevés, de l'ordre de $10^{-5} \mathrm{~A} \cdot \mathrm{cm}^{-2}$. Ainsi, afin de trouver l'origine de cette concentration importante de défauts dans la jonction nous allons étudier l'homogénéité du traitement laser. La technique de caractérisation LBIC a été utilisée dans le but de localiser des zones de forte concentration en défauts. En effet, ces derniers sont des centres de recombinaison pour les photo-porteurs, ce qui va se traduire par une baisse du photo-courant. La procédure de préparation des échantillons est décrite par la figure 3(a).
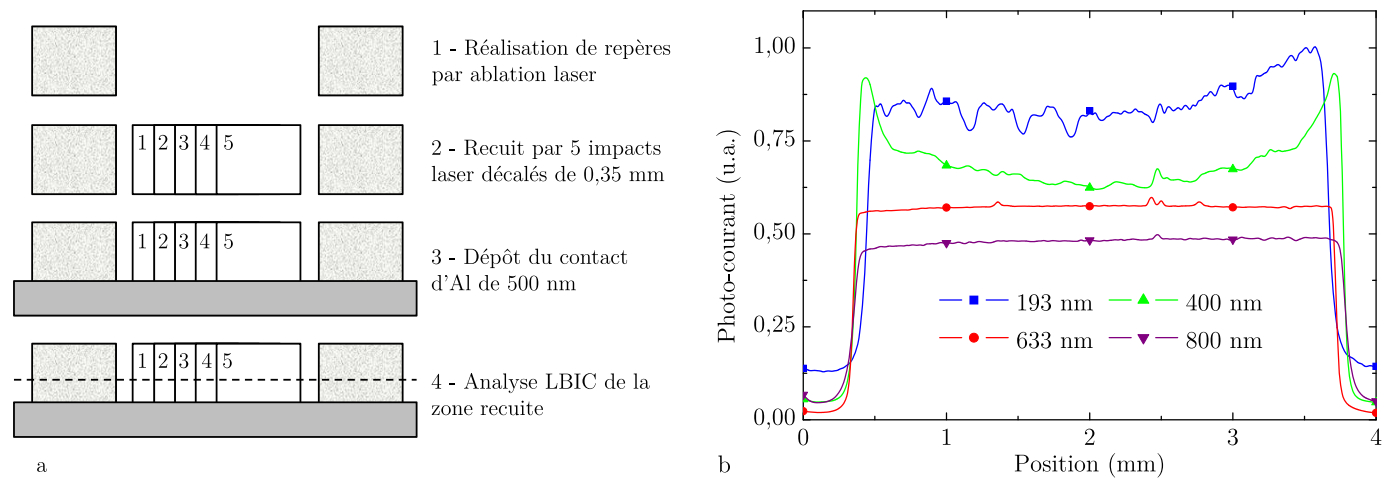

Figure 3. a) Procédure de réalisation des échantillons - b) Photo-courant mesuré en fonction de la position sur la région recuite pour différentes sources d'illumination.

Les mesures de photo-courant en fonction de la position sur l'échantillon, pour les quatre longueurs d'onde d'analyse, sont présentées sur la figure 3(b). Les profils obtenus á 800 et $633 \mathrm{~nm}$ sont relativement plats et homogènes. A $400 \mathrm{~nm}$, les bords de la zone recuite sont marqués par une forte augmentation, surement dûe á un effet de bord que nous ne pouvons pas expliquer, mais pas de variation caractéristique de défauts. Lorsque l'on réalise l'analyse á $193 \mathrm{~nm}$, quatre pics distincts de diminution du photo-courant de forme et d'amplitude identique apparaissent. De plus, l'espacement entre ces pics $(0,35 \mathrm{~mm})$ est identique á la distance séparant un impact laser d'un autre. Grâce á sa faible profondeur de pénétration dans le silicium, la longueur d'onde de $193 \mathrm{~nm}$ nous a permis de mettre en évidence que des défauts sont générés dans les premiers nanomètres de la jonction et sur les bords du faisceau laser. 

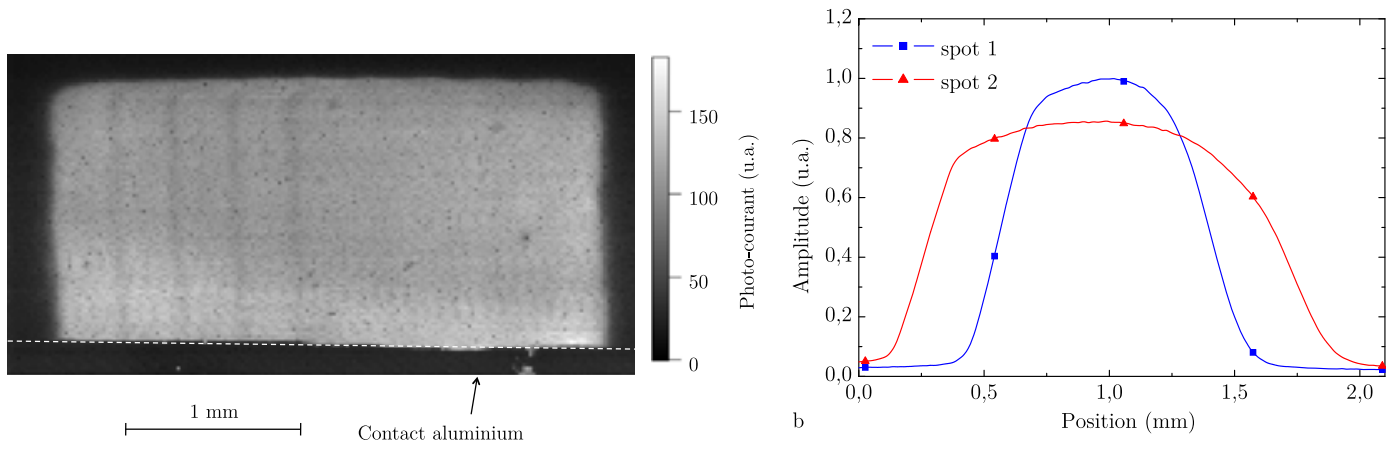

Figure 4. a) Cartographie du photo-courant sur une région recuite - b : Profil spatial des deux mises en forme de faisceau.

Afin de s'assurer de l'effet du bord de faisceau, une cartographie réalisée á $193 \mathrm{~nm}$ est présentée en figure 4(a). Les traces des d'impacts sont bien visibles. On peut supposer que les défauts sont générés sur tous les bords de faisceau. Cependant, seules les traces qui ne sont pas irradiées une seconde fois sont visibles. On peut conclure que le dernier impact guérit les défauts présents sous la zone irradiée.

Pour étudier l'impact du bord d'impact laser, nous avons réalisé des faisceaux avec des profils asymétriques présentés sur la figure 4(b). L'utilisation de profils de faisceau présentant des bords plus ou moins abrupts nous permet d'étudier l'effet des gradients d'énergie, et donc thermiques, sur la génération de défauts.

Nous avons vu que le dernier impact corrige les défauts présents dans la zone d'irradiation, masquant alors l'effet d'un des bords du faisceau. Pour étudier l'impact des deux profils de faisceau, il sera nécessaire de réaliser un déplacement de l'impact de recuit dans deux directions : une première de gauche á droite, permettant d'étudier le bord gauche du faisceau et réciproquement. L'analyse de l'impact du bord gauche est présentée sur la figure 5(a). Seules quelques faibles variations sont présentes pour les deux mises en forme de faisceau. Les défauts générés par ce type de profil sont suffisamment discrets pour ne pas impacter le photo-courant. Sur la figure 5(b) se trouve l'analyse du bord droit du faisceau. Celle-ci présente de fortes variations traduisant une importante concentration en défauts. Si le gradient énergétique de l'impact laser joue un rôle dans la génération de défauts, il n'est cependant pas l'acteur principal. En effet, le bord droit du spot 1 á un gradient compris entre celui du bord gauche du spot 2 et celui du bord gauche du spot 1, qui eux ne génèrent pas d'inhomogénéités. Cependant, un profil
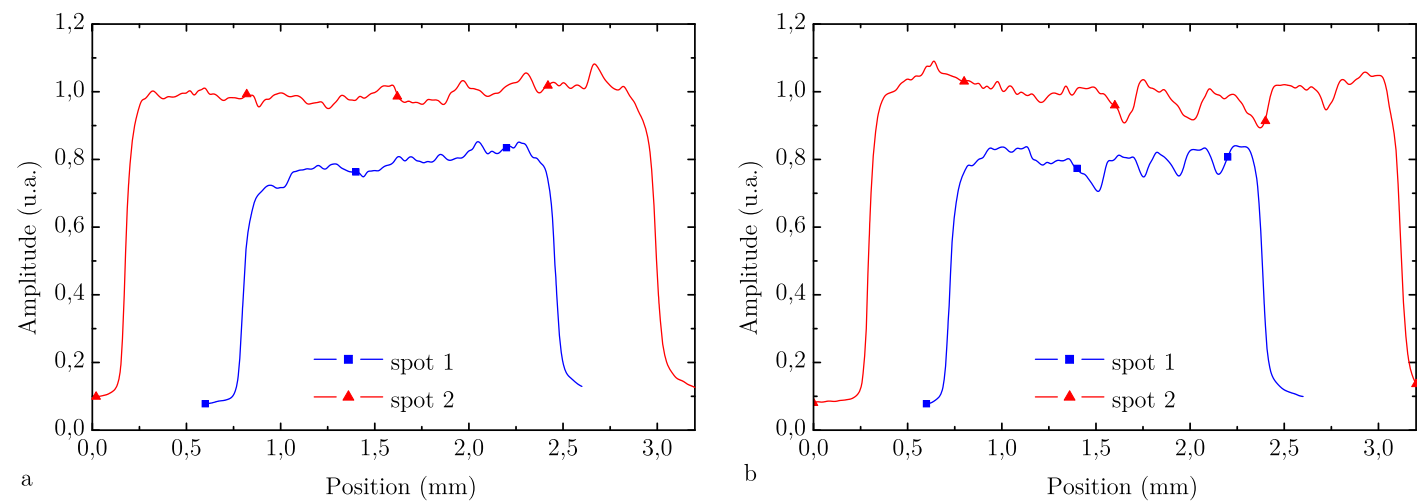

Figure 5. Photo-courant mesuré en fonction de la position sur la région recuite pour les deux mises en forme de faisceau : a) étude du profil gauche du faisceau - b) étude du profil droit du faisceau. 
de faisceau de forme courbe (bord droit) génère des défauts qui impactent fortement le signal d'analyse en comparaison avec un profil rectiligne (bord gauche). On peut supposer qu'un faisceau "Top Hat" serait idéal pour limiter ce phénomène, et ainsi obtenir un traitement plus homogène.

L'utilisation du LBIC nous a permis de repérer les zones où sont localisés les défauts. Nous avons découpé par FIB deux lames de quelques micromètres de profondeur : l'une dans une région présentant des défauts et l'autre dans une région sans défauts. Un microscope électronique á transmission haute résolution a permis d'obtenir les images présentées en figure 6. Alors que la structure cristalline d'une zone sans défaut est parfaite, avec une surface monoatomique, celle d'une région présentant des défauts est fortement perturbée, avec une déformation du réseau cristallin en surface ainsi que la présence de fortes contraintes dans le matériau. Le fait que cette perturbation s'étende sur une distance de seulement quelques nanomètres explique le fait que seule une longueur d'onde fortement absorbée dans le matériau permette de détecter ces défauts.

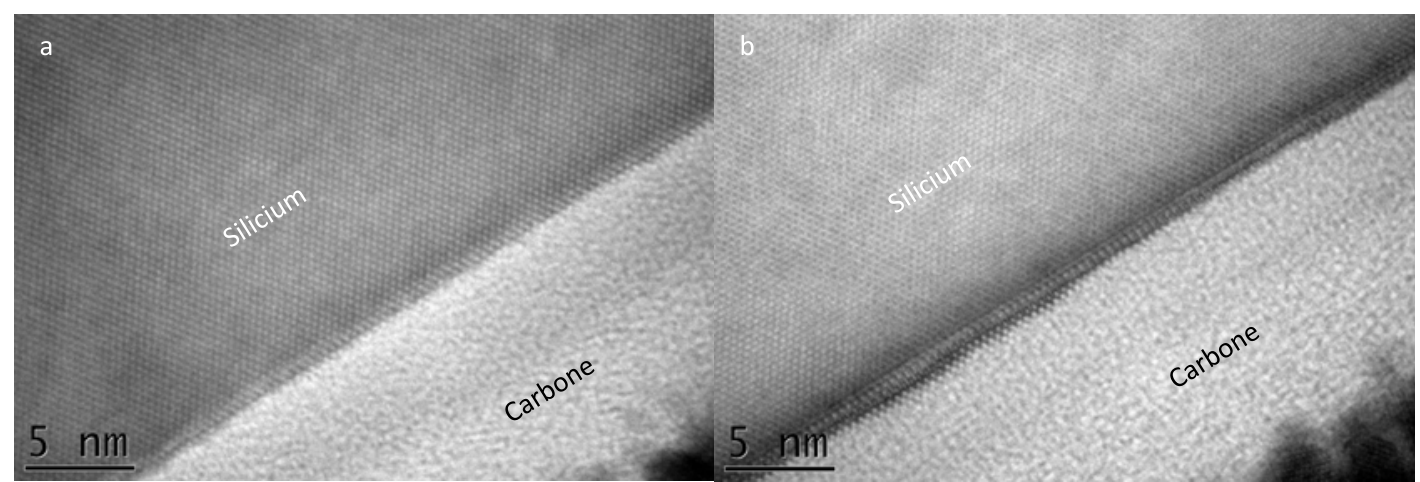

Figure 6. Image TEM : a) coupe réalisée au centre d'un impact laser - b) coupe réalisée au bord d'un impact laser.

\section{CONCLUSION}

L'association d'une implantation par immersion plasma et d'un recuit laser permet l'obtention de

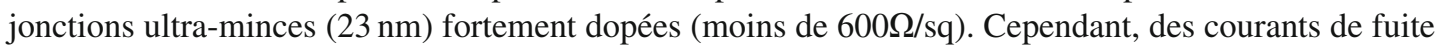
de $10^{-5} \mathrm{~A} \cdot \mathrm{cm}^{-2}$ traduisent la présence d'une concentration de défauts dans la jonction trop importante.

L'utilisation d'un dispositif LBIC nous a permis de mettre en évidence des inhomogénéités localisées sur les bords d'un impact laser. Ces inhomogénéités se révèlent être des défauts de cristallographie (déformation du réseau et contraintes) du silicium présents sur une épaisseur de $3 \mathrm{~nm}$.

Cette étude reste á compléter afin de réduire voire d'éliminer ce problème d'inhomogénéité.

\section{Remerciements}

Ce travail est réalisé dans le cadre du projet ALDIP (Activation Laser de Dopants implantés par Immersion Plasma), financé par le Conseil Général 13, la région PACA et la Direction Générale des Entreprises. Nous remercions la plateforme CIMPACA pour les analyses SIMS.

\section{Références}

[1] F. Torregrossa et al., Proc. 16th International Conference on Ion Implant Technology, (2006) 6

[2] V. Vervisch, Thèse de 3ème cycle (Etude et realization de jonctions untra fines $\mathrm{p}+\mathrm{n}$ par la technique d'implantation d'ions par immersion plasma. Application aux cellules photovoltaicques) (2007)

[3] F. Torregrosa et al., Surf. and Coat. Tech., 186 (2004) 93-98 
[4] J. Venturini et al., Thin Solid Films, 453-454 (2004) 145-149

[5] G. Fortunato et al., Nucl. Instr. and Meth. in Phys. Res. B, 186 (2002) 401-408

[6] M. Hernandez et al., Appl. Surf. Sci., 208-209 (2003) 345-351

[7] G. Kerrien et al., Appl. Surf. Sci., 208-209 (2003) 277-284

[8] N.M. Thantsha et al., E. E. van Dyk, Physica B, 2404 (2009) 4445-4448

[9] V. Sirotkin et al., Materials Science and Engineering B, 91-92 (2002) 260-263

[10] P. Vorasayan et al., Solar Energy Materials \& Solar Cells, 93 (2009) 917-921

[11] I. Périchaud et al., Materials Science and Engineering B, 42 (1996) 265-269

[12] ITRS International Technology Roadmap for Semiconductor (2009) www.itrs.net

[13] A. Florakis et al., Nucl. Instr. and Meth. in Phys. Res. B, 253 (2006) 13-17

[14] F. Torregrosa et al., Nucl. Instr. and Meth. in Phys. Res. B, 237 (2005) 18-24

[15] V. Vervisch et al., Appl. Surf. Sci., 255 (2009) 5647-5650

[16] CRC press 85th edition, 12-150 (Handbook of Chemistry and Physics) (2004-2005) 